\title{
IVD.20 - Development of Quantitative Real-Time PCR (TaqMan Triplex System) for the Diagnosis and Evaluation of Therapeutic Efficacy in Chagas Disease
}

Constança Britto $^{1}$; Carolina Messias ${ }^{1 *}$; Otacilio Moreira ${ }^{1}$; Antonio Gomes Pinto Ferreira ${ }^{1}$; Raouf Emile Sykora ${ }^{1}$; Marco Aurelio Krieger ${ }^{1}$; Alexandre Costa ${ }^{2}$; Alice Gomes ${ }^{1}$; Jéssica Constermani ${ }^{1}$.

1Fiocruz - Fundação Oswaldo Cruz;

2IBMP- Instituto de Biologia Molecular do Paraná.

Introduction: Chagas disease (CD) affects about 8 million individuals in endemic areas with 20 to $30 \%$ eventually developing chronic Chagas cardiomyopathy, the most severe manifestation of the disease in humans. Serology is the gold standard for the diagnosis of chronic CD, whereas conventional and quantitative Real Time PCR (qPCR) have been used as complementary tools for diagnosis in cases of congenital transmission, discordant serological results in chronic patients and for evaluating etiological treatment.

Objective: Based on the proof of concept previously generated by our group for developing a prototype qPCR duplex diagnostic kit (T. cruzi nuclear satellite DNA [SAT] and a heterologous internal amplification control [IAC]), we propose here the addition of a second target [kDNA] for T. cruzi detection in a single reaction (TaqMan triplex qPCR) to improve the sensitivity of the test.

Methodology: The first steps for standardization consisted in defining the best concentration of primers and probes (SAT DNA: 900nM of each primer, 150nM probe; kDNA: 300nM of each primer, $50 \mathrm{nM}$ probe; IAC: $200 \mathrm{nM}$ of each primer, $50 \mathrm{nM}$ probe), and the adjustment of the standard curves with the use of synthetic DNA for the three targets, to reach better accuracy in quantifying parasite load.

Results: Our results demonstrated optimal identities for sequences obtained from the amplified products generated by each target (96\% kDNA, 97\% SAT DNA, 100\% IAC) when compared to those described in the database, and these sequences were already synthesized. Preliminary tests with the synthesized sequences showed a linearity of $10^{7}$ to $10^{2} \mathrm{DNA}$ copies.

Conclusion: Standardization will also involve the testing of linearity, inclusiveness, exclusivity, precision, limit of detection (LOD), limit of quantification (LOQ) and clinical sensitivity and specificity of the reaction. We have as perspectives the setting of the triplex reaction using reagents produced by IBMP/Fiocruz and to validate the assay with a panel of blood samples from patients with chronic CD. This project is a partnership between IOC and BioManguinhos, and has recently received financial support from the INOVABIO program.

Keywords: Chagas disease; Real Time PCR; Trypanosoma cruzi 